\title{
LE RECUEIL LATIN DES APOPHTEGMES
}

On ne s'est guère occupé, depuis la magistrale publication du jésuite Rosweyde ${ }^{\mathrm{I}}$, des collections latines qui correspondent aux livres V et VI des Vitae Patrum ${ }^{2}$. Il reste encore beaucoup à dire sur les manuscrits, en attendant qu'un jeune courage entreprenne, à partir des documents grecs, syriaques, coptes et arabes, la reconstruction de tout le recueil 3. Dans ce travail, les collections latines fourniraient un point de comparaison fort utile.

L'examen de divers manuscrits latins de cette espèce m'a permis de reconnaitre un petit groupe de «sentences * ou apophtegmes monastiques - sententiae patrum - qui n'ont point encore rencontré d'éditeur. Elles sont au nombre de trente-six, très courtes, et anonymes ; introduites, pour la plupart, par les mots : Ait senex, Inquisitus senex... respondit, Aiebant senes, ou un équivalent. Ce double caractère, extrême brièveté et anonymat, suffit à donner au groupe une physionomie à part dans la vaste littérature des apoph. tegmes. Ceux-ci sont habituellement attribués à un personnage déterminé, plus ou moins célèbre, et, tout en variant de longueur, possèdent quelque étendue. Les Sentences sont, je le répète, des maximes d'une rara sobriété, la concentration d'une pensée spiri-

1. Vitae Patrum. -. De vita et verbis senionum libri $X$ historiam eremiticam complectentes. Opera et studio Heriberti Rosweydi, Anvers 1615 (puis Lyon 1617) ; nouvelle Édition revisée en 1628. Sur l'importance de cet ouvrage, voir les récentes remarques du P.Hippolyte DeLEHAYE dans A traters trois siècles : l'aurre des Bollandistes 1615-1915, Bruxelles 1920, p. 7-22 ; on notera particulièrement ces jugements délibérés du président des modernes bollandistes: ( ... cette aurore du bollandisme que fut la Vie des Pères $)$ (p. 2); a c'est véritablement la pierre fondamentale des Acta sanctorum $)$ (p. 16).

2. Je renvoie à la rédition comprise dan

3. Parmi les plus récentes études, soit de simple orientation soit de critique, sur ce sujet, je citerais : C. BUTLER, The Lausiae History of Palladius, I, 1898, p. 208-214; \$. Vailhé, dans Échos d'Orient, V, 1901, p. 39-46; M. Chaine, dans Mélanges de la Faculté Orientale de Beyrouth, $\mathrm{T}, 1912$, p. $541-569 ; \mathrm{F}$. NAU, dans I'erue de l'Orient ohrétien, XVIII, 1913, p. 208-212 ; P. NIKITIN, dans Fiz. V'emenik, 1915-1916, p. 127. 176 (dissertation en russe sur a le Paterikon grec de Sceté $n$, cf. Byzantinische Zeitschrift, XXIII, 1914-1919, p. 455); A. H. SALONIUs, Vitae patrum, Lund, 1920. Parallèlement, on devra tenir compte des textes qui accroissent désormais les matériaux déjà réunis par Rosweyde, Cotelier et Zoega : avant tout, la collection syriaque d'A nan.Isho, Editée par le P. BEDJAN (Paris 1897), et de nouveau, arec traduction anglaise, par W. BuDge (Londres 1904); et la collection grecque du manuscrit Coislin 126 de la Bibliothèque Nationale, partiellement imprimée et traduite par F. NAU dans la Rerue de l'Orient Chrétien de 1907 \& 1913 (volumes XII.XVIII).

Revue Búnédictine. 
tuelle qui ne redoute pas de paraitre elliptique, si elle n'est pas toujours vraiment profonde. Eu égard au classement de cette littérature, il est naturel de supposer qu'elles représentent un résidu ; tout le reste ayant été disposé en catégories didactiques et selon l'ordre alphabétique des noms des ascètes ' au bout du compte, avec un reliquat d'étroites formules qui ont ainsi formé, presque mécaniquement, une série supplémentaire et apparemment homogène ${ }^{2}$.

Après avoir discerné cet aspect des Sentences d'après leur place dans les collections latines, je n'avais aucun doute qu'on les dût retrouver en grec. Elles existent, en effet, substantiellement les mêmes et dans le même ordre, mais avec des interpolations plus ou moins graves, dans un certain nombre de manuscrits du fonds grec de la Bibliothèque Nationale, à Paris 3 . Mon dessein était de les faire connaître parallèlement en grec et en latin. M. l'Abbé Nau ayant publié un texte grec à peu près complet dans son analyse du ranuscrit Coislin r26 (X-XIe siècle) ${ }^{4}$, je me contenterai de donner la version latine, après avoir indiqué les témoins de cette tradition occidentale. Mais ceci ne peut aller sans appeler l'attention sur la

1. Nikitin (voir ci-dessus) a bien distingue les trois types des Paterika : alphabétique systématique, alphabétique-systématique. Ce dernier est précisément représenté par la grande collection des Adhortationes (livres V et VI de Rosweyde), comme il est aisé de Je constater ; l'alphabétisme, en particulier, est très clairement sauvegardé dans la recension latine. Le premier chapitre, par exemple, sur la vie de perfection (De profectu

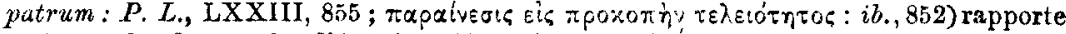
en bon ordre des paroles d'Antoine (A), Grégoire (T). Evagre (E), Zacharie (Z), ThEodore (8), Jean et Joseph (I), Cassien (K), Nesterôs (N, Pastor (pour Poemen) et Pambo

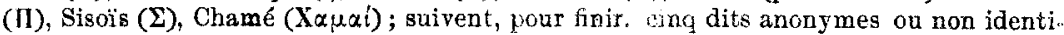
fiés : $n^{\circ 8}$ 19.23 (en réalité, les textes de Cotelier pernettent d'en identifier trois).

2. A part la brièveté constante des formules, elle est semblable aux séries anonymess qui achèvent, comme je viens de l'indiquer, chacune des cections des Adhortationes. ... Je note tout de suite qu'on retronve deux sentences ( ${ }^{\circ \mathrm{B}} \mathrm{XVI}$ et XXXII) dans le petic recueil qui porte le nom de Martin de Dumio (nos 92 et 93: P. L., LXXIV, 392), et une troisième $\left(\mathrm{n}^{\circ} \mathrm{XXXIV)}\right.$ dans le dernier chapitre des Adhotationes (n०22:P, $n_{\text {: }}$ LXXIII, 1019). Les différences de rédaction sont telles qu'il faús supposer des tradirs. teurs indépendants.

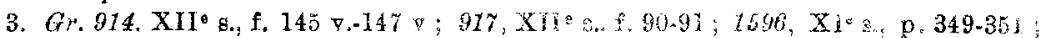

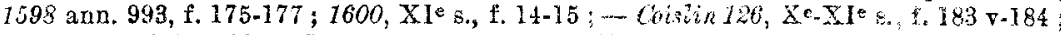

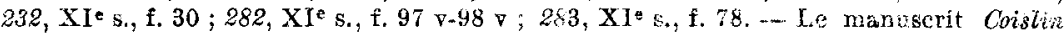
127, f. 308 , ne présente que le titre de la série originalı, peut-être par suitea'une lacune. Ce titre est donne par presque tous les manuscrits (point yar le Coislin 120 ): "A $\pi 0 \varphi \theta$ é $\gamma$ -

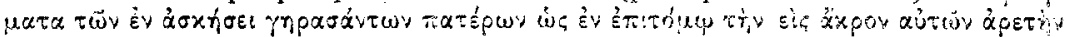
Sทุดoัvex.

4. Revue de l'Orient Chrétien, XII, 1907, p. 401-403, nou 83 i 123. ... Les sentences IV et XI manquent dans la rédaction propre a ce manuscrit. Par contre, il en donne une autre $\left(i b ., n^{\circ} 111\right)$, dont l'authenticité est asiurée par laccord des témoins de la tradition grecque. Celle-ci, en outre, débute normalement par trois dits anonymes ar les vices qui sont attribnéb ailleurs à labbé Isaie (cf. P. á, LXV, 181-184. no 9.11) 
ressemblance du contexte général de part et d'autre, et le sujet requiert quelque développement.

Les manuscrits latins qui comprennent des collections d'apophtegmes sont extrêmement nombreux dans les dépôts modernes et constituent une des sections littéraires les mieux fournies, à côté des bibles, des canons, des sacramentaires et des vies de saints. C'est sans doute cette abondance qui détourne de l'examen, outre l'apparente complexité de la matière. Si l'on y prend garde cependant, le compte des témoins anciens ou intacts n'est pas très long, dans la classe des Vitae Patrum; et cette classe est distribuée, de même, en catégories assez nettes, dès qu'on a recours aux exem. plaires typiques: vies proprement dites, récits historiques (du genrt: de l'Hisloire Lausiaque), apophtegmes.Ces derniers forment principalement un grand recueil qui porte d'ordinaire, d'après son premier chapitre, le titre d'Adhortationes patrum, et commence par ces mots significatifs: «Interrogauit quidam abbatem Antonium, dicens : Quid custodiens placebo Deo? Rosweyde, pour garder le plan des premières éditions ${ }^{\mathrm{I}}$, en a fait ses $\mathrm{V}^{\mathrm{e}}$ et $\mathrm{VI}^{\mathrm{e}}$ livres, et le tout est bien connu, à peu de chose près; mais on a perdu de vue le cadre primitif, à force de transcrire ou d'imprimer ce riche répertoire. Il est donc indispensable, tout d'abord, d'en reproduire la physionomie certaine. Voici, en effet, comment l'ensemble nous a été réellement livré. J'indiquerai plus loin mes sources et les points d'appui de la tradition grecque.

I. Adhortationes sanctorum patrum ad profectum perfectionis monachorum (Rosweyde v-I).

II. De eo quod oporteat quidem omni festinatione sectari (Roszeyde v-2).

III. De compunctione (Rosweyde v-3).

IV. De continentia et quia non solum in cibis sit adsumenda continentia sed etiam in ceteris animae motibus (Roszeyde v-4).

1. Les éditions elles-mêmes suivent l'usage des manuscrits de la fin du moyen âge. Je remarque déjà le partage des Adhortationes en dix-huit « livres " (Pélage) et quatre (Jean) dans le manuscrit de Troyes 716 (Bouhier), XII e siècle (fol, 1-108). D'autre part, le plan d'ensemble de l'ouvrage de Rosweyde apparaît dan un autre manuscrit de Troyes, $\mathrm{n}^{0} 655$ (Pithou), de même XIl* siècle (fol. 1-197); après les Vies d'Antoine, Hilarion, Basile, Malchus, on a successivement les livres désignés par hosweyde: II (Historia Monacĭorum), III (Verba seniorum), IV (extraits de Sulpice Sérère et de Cassien), V.VI (Ṕllage-Jean, mais ici incomplètement). On peut comparer les trois ancieanes éditions typiques que décrit Rosweyde (Prolegom, XVITT-XX:P, LXXIII, 64 ss.) 
V. Relationes diuersae pro cautela quae adhibenda nobis est contra insurgentia in nobis fornicationum bella (Rosweyde $y_{-5}$ ).

VI. De non possidendo in quo oportet cupiditatem uitari (Rosweyde v-6).

VII. Narrationes diuersae ad patientiam et fortitudinem nos aptantes (Rosweyde v-7).

VIII. De eo ut nihil per ostentationem fieri debeat (Rosweyde v-8).

IX. De eo quod non oporteat iudicare quemquam (Rosweyde v-q).

$\mathrm{X}$. De discretione (Rosweyde v-ro).

XI. De eo quod oporteat semper sobrie uiuere (Rosweyde $7-I I$ ).

XII. De eo ut sine intermissione et sobrie debeat orare (Rosweyde v-I2).

XIII. De eo quod oporteat hospitalem esse et musericordem ir hilaritate (Rosweyde v-I3).

XIV, De oboedientia (Rosweyde $\cdots-14$ )

XV. De humilitate (Rosweyde v-15)

XVI. De patientia (Rosueyde v-ro).

XVIY. De caritate (Rosweyde $\eta^{\prime} \cdot I 7$ ).

XVIII. De praeuidentia siue de contemplatione (Rosweyde $v$ - $1 f$ et vi-L). - Au point où commence Roszueyde vi-I, les manuscrits insirent cette note: Vsque hic de greco in latinum transtulit Pelagius diaconus ecclesiae romanae. Subdita Iohannes subdiaconus.

XIX. De sanctis senioribus qui signa faciebant (Roszeyde vi-z).

$\mathrm{XX}$. De conuersatione optima diuersorum sanctorum patrum (Rosweyde vi-3).

XXI. Septem capitula uerborum quae misit abbas Moyses abbati Pemenio et qui custodierit ea liberabitur a poena (Rosweyae vi-4). - Au terme du texte imprime par Rosweyde (\$ 37), on lit cette nouvelle souscription: Expliciunt sermones sanctorum patrum interpretati de greco in latinum a diacono Pelagio sanctae ecclesiae romanae et Iohanne subdiacono.

XXII. Correctiones patrum (Rosweyde viö \&4).

XXIII. Sententiae patrum (texte snédit).

1. Ce tirre est le seul qui donne liea à des varianies. Ainsi hubin, 1 accorde, je crois les principaux témoins. Toutefois, je me demande si la rédacion primitive r'offrait pas, au singulier, adhortatio ( $\pi \alpha p x i v a \sigma s)$, comme ci-dessous dans le titre de la collection di Saint-Médard. La suite est exacternent conforme au titre grec (voir par exemple Cois!. 127, fol. $6^{\mathrm{v}}$, et comparer l'analyse de Photius, $P . L .$, LXXIII, $852 b$ ); seul, le mot monachorum serait de trop. - Pour en venir au détail, ce titre précis est attesté par les collections de Saint-Martial, d'Albi et de Montmajour (à part, dans cette dernière, de légères modifications : exortatio is, profectionis). On a ensuite : Bobbio (qui omet sanctornm et perfectionis); Saint-Médard (de même, en outre: liber sermonum usl adhortationis, ce dernier mot n'étant peut-étre qu'un pluriel déguisé); Wurzbourg (au lieu de perfectionis, perfectorum); Saint-Florian (le seul des manuscrits anciens qui attribue à s. Jérôme: adorta( (iones?) sanciorum, fatrum perfec(torum?) monnchorum quas de greco in latizun. transtulit beatus hieronimus $p b r)$. Cluny glose sous cette forme: adhortationes siue exempla beatorum pairum ad profeclum et institutionem monachorum huic seculo perfecte abrenuntiantium. La collection wisigothique, malheureusement, fait défaut.

II. J'ai désormais pour témoins or iiaaires du texte : Cluny (arec lequel concourt Sainto 
Martial), Saint-Médard (avec lequel concourt Albi), Saint-Florian, Bobbio (imparfaitement connu); la collection wisigothique n'est disponible qu'à partir du quatrième titre. Saint-Médard (Albi) : Catitulum eo quod; Saint-Florian: oportet (cf. le grec), et omni

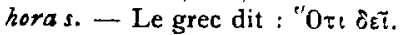

III. Photius a omis ce chapitre; il est dès lors en retard d'un chiffre.

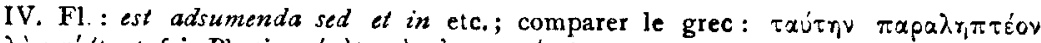

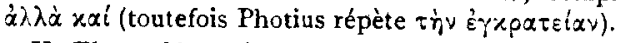

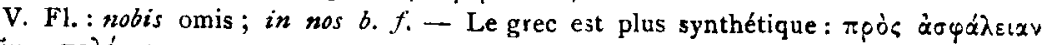
$\tau \tilde{\omega} v \ldots \pi, \ldots \lambda \varepsilon \mu \omega \nu$.

VI. Med. : Capitulum de n. ; quod op. et. Wis : possidendum ; uitari. - Grec : हैे $\bar{\psi}$

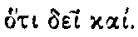

VII. Med. : paenitentiam; de même, F1., qui remplace aussi aptantes par coortantes.

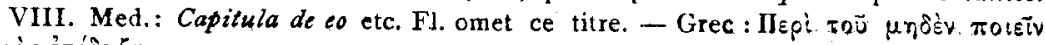

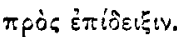

IX. Fl. : oportet. Wis. : indagari.

XI. Med.: Capitula de eo quia. Cl.: oportel.. FI. omet ce titre. - Grec: vńpetv.

XII. Bob., Med., Fl. : Capitulum ut ; le grec a тврі тoú et l'infinitif. Fl., de plus, omet et sobrie.

XIII. Bob.: Quod oporteat; Fl.: Quod oportet; Med.: Capitulum quod oporteat

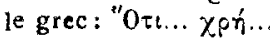

XIV. Med. prépose : Capitulum, et de même à l'article suivant ; ensuite, il fait défaut, ainsi que Fl. - Wis. ajoute : cum timore custo.lienda atque serwanda.

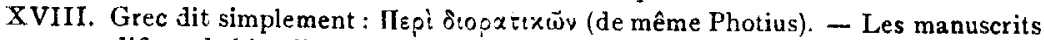
récents modifient habituellement la souscription: Vsque huc ou Hucusque... abhinc deorsum lohannes, ou simplement abhinc autem, abhinc (par exemple B. N. 17623, B. M. 38684, Châlons 54, Rouen 7375 , Troyes 716 ). - C'est cette note, très probablement, qui a suggéré l'attribution du recueil à s. Jérôme. Les mots « Pelagius diaconus ecclesiae romanae \, qui évoquaient presque fatalement le souvenir du moine breton, durent parâitre incroyables $\$$ quelque ancien éditeur, et la mention * Hieronymus presbyter $\gg$ beau. coup plus vraisemblable. On rapprochera notamment le titre, déjà rappelé, du manuscrit de Saint-Elorian et celui du manuscrit de Valfroy (B. M. 38684 , fol. 95) : Capitula adhortationum sanctorum quas de greco in latinum transtulit Pelagius diaconus ecclesiae romanae,

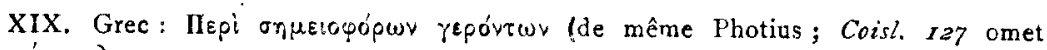

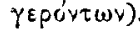

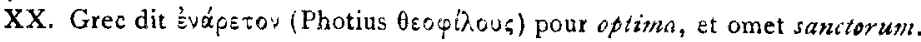

XXI. Wis. omet septem, mot confirmé par le grec; mais celui-ci omet uerborum.

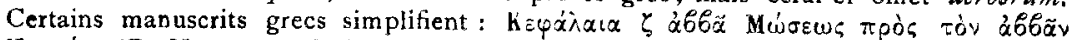
Ilolúvva (B. N. gr. ${ }^{5} 598$, fol. J77). La remarque qui suit est plus développée: $x \alpha i$ ó

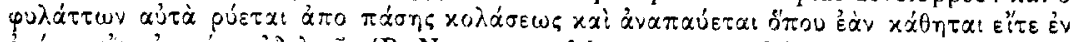

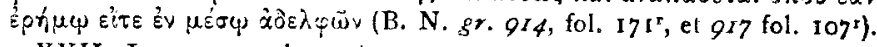

XXII. Les manuscrits préposent naturellement: Incipizunt; de même, à l'article suivant. - Dans le grec, ce morceau complète le livre XX (Rosweyde VI, 3) : il manque souvent de titre ou bien est désigné simplement: IIEpi $\tau \tilde{\omega} \vee \delta \omega \delta \varepsilon \times \alpha \alpha_{\alpha} \vee \alpha_{\chi} \omega p_{1} \tau \tilde{\omega} \vee$ (voir cependant $P$. L., LXXIII, I062, n. 5).

XXIII. Cluny ajoute sanctorum. Pour le grec, voir ci-dessus. - Cluny et Saint-Martial ont un explicit dont l'authenticité est contestable: Expliciunt sententiae de diuersis patribus collectae.

Il s'en faut de beaucoup, malheureusement, que tout ce recueil soit représenté par des exemplaires très anciens. Nos ressources sont minces, à cet égard; et le plus étrange, à première vue du moins, est que l'immense succès de cette littérature d'édification est précisément responsable de la disparition presque complète des 
collections formées au début du moyen âge, avant le IXe siècle ${ }^{\text {I }}$. Les catalogues suffisent à nous donner une idée de la diminution de notre héritage. Les copies se succédant habituellement les unes aux autres pour des motifs d'ordre pratique, les plus anciennes étaient destinées à périr, à moins de chances extraordinaires. Le même phénomène se laisse observer dans tout le champ de l'hagiographie ${ }^{2}$. Ainsi en est-il également pour les livres liturgiques et les volumes de Règles. Si les manuscrits de la Bible et des conciles ont moins souffert, relativement, de l'action du temps, c'est pour des raisons qu'il est aisé d'apercevoir. Compte tenu des exceptions, l'histoire des bibliothèques médiévales est dominée par ce simple fait qui n'offre rien de mystérieux et dont on pourrait tirer nombre de conséquences : les vieux livres ont été éliminés et remplacés normalement pour satisfaire aux besoins des nouvelles générations ${ }^{3}$.

Cinq manuscrits des Adhortationes, à ma connaissance, sont antérieurs au IXe siècle. Mais aucun n'est complet. Le plus satisfaisant (son étendue étant certaine), celui de Bobbio $\left(\mathrm{n}^{0}{ }_{5}\right)$, ne donne qu'une recension abrégée. De plus, les dates supposées des manuscrits de Cologne et de Wurzbourg sont sujettes à caution. Je les range selon leur importance littéraire.

(r.) Londres, B. M. Add. $1535^{\circ}$ : deux feuillets doubles (fol. I et $\mathrm{I} 2 \mathrm{I}$ ), écrits en une onciale encore naturelle qui peut être de la fin de VIIe siècle ou du commencement du VIII ${ }^{4}$; ces fragments qui forment la couverture d'un cartulaire du XIIe siècle proviennent de la cathédrale de Winchester et restituent deux morceaux des livres XIII et XIV : à savoir XIII $\$ 9-X I V \S I$ et $\mathrm{XIV} \S \mathrm{ro-I} 7$.

1. Le recueil des « vies des Pères » est déjà mentionné par Cassiodore, De institutione divin. litterarum, c. $32(P . L, L X X, 1147 d)$; de même, une référence précise dans le Decretum de đGélase 》, IV, 4, 2 (éd. Dobschuetz, 1912, p. 42, et cf. p. 275).

2. On n'ignore pas combien sont peu nombreux les recueils de vies de saints antérieurs an IX ${ }^{\circ}$ siecle (cF. Analecta Bollandiana, XXIX, 1910, p. 5 ss.). Ceux même qu'on attribue, arec plus ou moins de raison, an VIII e siècle, sont, je crois, de la période carolingienne.

3. Ceci n'empeche ni que les gens du moven âge aient pris grand soin de leurs livres, ni non plus que nos pertes sient été beaucoup accrues par suite des incendies, des guerres ou du vandalisme moderne. - Je ne puis manquer cette occasion de rappeler au lecteur les spirituels a essais de S. R. MAITLAND, intitulés The dark Ages (1844 et 1845, 3. éd. 1888). Ce sont des pages virantes et qui n'ont pas vieilli, les plus persuasives peut-être qui furent jamais écrites sur la culture littéraire du moyen âge, le respect qu'on professait alors pour le livre, les difficultes qu'on dut surmonter pour transmettre le flambeau. L'enthousiasme intelligent de Maitland est d'ailleurs soutenu par une connaissance remarquable du passé, histoire et littérature.

4. Cf. Catalogue of anoients Manusoripts in the British Museum, 20 partie, 1884, p. 62 . 
(2.) Cologne, Chapitre 16.5 , I 8 feuillets, en une semionciale qui serait du VIe ou du VIIe siècle ${ }^{\mathbf{r}}$ : les dix premiers livres, jusqu'à $\mathrm{X} \S 68$, sans titres ni divisions.

(3.) Bruxelles, B. Royale I22I (9850-52), fol. 5-107 : l'un des témoins du texte de Rosweyde, manuscrit oncial fort connu, daté 695-7 I I et localisé à Saint-Médard de Soissons ${ }^{2}$ : livres I-XV $\$ 39^{3}$.

(4.) Wurzbourg, Université, Mp. th. qu. 3o, 76 feuillets : ancien manuscrit de la Cathédrale en minuscule anglo-saxonne du VIIIe siècle ${ }^{4}$ : livres I-XXI $\S 4^{5}$.

(5.) Milan, Ambrosienne $F .84$ sup., I 53 feuillets : manuscrit de Bobbio rédigé en onciale vers le début du VIIIe siècle : extraits des livres I-XXI, suivis du livre XXIII 6 .

Le IX ${ }^{e}$ siècle a produit plusieurs manuscrits qui nous ont été conservés. Le plus célèbre est la collection de Saint-Florian (Bruxelles $8216-8218$, fol. $62^{\mathrm{r}}-148^{\mathrm{v}}$ ), employée encore par Rosweyde ${ }^{7}$ et datée de l'an $819^{8}$. Il prend fin au livre XV $\$ 16{ }^{9}$.

Ensuite les matériaux abondent, mais de différente qualité. Il vaudrait peut-être la peine d'en faire l'inventaire complet. J'ai distingué trois collections qui, rejoignant le manuscrit de Bobbio, d'une part, et, de l'autre, la tradition grecque, permettent de délimiter l'authentique recueil des apophtegmes dans sa forme latine. Il doit en subsister d'autres encore, semblables. Celles-ci suffiront au présent dessein :

(I.) Paris B. N. 5387, I9 I feuillets $10(30 \times 20)$ : manuscrit de

1. Cf. Ph. JAFFÉ et W. WAтtgMBaCh. Eeclesiae metropolitanae Coloniensis codices manusoripti, 1874, p. 65 .

2. Voir la bibliographie rassemblée par P. LehMann ap. L. Traube, Forlesungen und Abhandlungen, I, 1909, p. 178 ( $\left.\mathrm{n}^{\circ} 27\right)$; ajouter: PrThd, Analecta sacra, II, 1884, p. 624-634, 649 sq., et facs. no 2 ; New Palaeographical Socisty, $2^{\circ}$ вérie, 1904, pl, 28-29; Revue bénédictine, XXII, 1905, p. 12 ss.; F. STEFFens, Lateinische Paläographie, $2^{\circ}$ ed. 1909 , pl. 29 a.

3. L'influence de cette collection incómplète paraît avoir été grande. Je la retrouve exactement, au même point d'arrêt, dans B. N. 9729 (fol. $82^{r}-153^{\mathrm{r}}$ ), IX siècle, de Saint-Martin de Tours (primitivement terminé a XV $\$ 21$ ), et $A c q .697$ (fol. 158 $\left.8^{\vee}-242\right)^{4}$, XII' siècle, du Chapitre d'Albi.

4. Cf. J. A. OEGG, Versuch einer Korographie ron Würzburg, I, 1808, p. 440-443; A. Pongelet, Analecta Bollandiana, 1913, p. $425 \mathrm{sq}$.

5. C'est-à-dire Rosweyde VI, $4 \$ 4$.

6. Voir la description d'A. RetpeEsscherd, Bibliotheca patrum latinorum italica, II, 1871, p. 97 sq. ; E. ChatelaIN, Unoialis seriptura, 1901. pl. XLI, 1; E. H. ZIM. MERMAN, Vorkarolingische Miniaturen, 1916, pl. 17 et 18 .

7. Prolegomonon $X X I V: P . L$., LXXIII, $80 a$.

8. Cf. New Palaeographical Society, 2e série, 1904, pl. 31.

9. J'indiquerais encore pour cette époque : Chartres 5 , fol. 196-243 (collection brouillee) ; Orléans 338, fol. 275-290 (extraits en désordre) ; B. N. 9729 (voir plus haut)

10. En fait, la numérotation moderne a laissé hors de compte huit feuillets : après 7 , $43,73,134,135,148,179$ respectivement. A l'état complet, le manuscrit devait aroir 208 
Colbert, assigné, sans autre remarque, au IX' siècle par le catalogue de 1744 et de même, récemment, par les Bollandistes I. Je n'ai pas été peu surpris de constater que ce beau volume, privé seulement de son premier cahier, était, d'un terme à l'autre, l'œuvre d'une main wisigothique fort experte ${ }^{2}$. Il a pu d'ailleurs être écrit en deçà des Pyrénées, en quelque lieu de la province de Narbonne. Diverses notes de la dernière page (fol. $189^{r}$ ), maintenant presque illisibles ${ }^{3}$, favoriseraient cette hypothèse; car on y retrouve le style continental du $\mathrm{X}^{\mathrm{e}}$ siècle. Mais l'aspect ne diffère pas sensiblement de celui des produits authentiques de l'école de Tolède. Rapproché des modèles réunis par Ewald et Loewe ${ }^{4}$, sa date paraît être la fin du IXe siècle. Il reproduit exactement, lacunes à part, le recueil que j'ai présenté; dans son état actuel en effet, il est privé des livres I et II, par défaut du cahier initial, et, à la fin, du texte des vingt-quatre premières Sentences, par l'arrachement d'un feuillet (entre I 88 et I89) 5. En dépit de ces pertes, la collection wisigothique de la Bibliothèque Nationale, homogène et nette, reste notre meilleure garantie pour l'établissement du recueil latin des apophtegmes; j'oserai même avouer que, sans son secours, toute cette question littéraire m'aurait échappé.

(2.) Paris B. N. Acq. I49I : manuscrit clunisien de plus de huit cents pages, « un très remarquable monument de l'art des enlumineurs du XIe siècle ${ }^{6}$ et, en même temps, un répertoire complet d'ascèse monastique, comprenant, outre le recueil des $A$ dhortationes

feuillets, distribués en vingt-six cahiers (signés). - Les deux derniers du compte actuel sont étrangers au manuscrit ; ils ont été empruntes à un antiphonaire de la messe en notation a quitaine du XI' siècle (fol. J10 : partie du trai "Qui habitat in adiutorio altissimi 》 pour le premier dimanche du carême; fol. 191: messe \& Esto mihi D. R. "Iubilate domino", Of. "Benedictus es domine" a vee les versets, Co. « Manducar verunt $n$ ).

1. Catalogus codicum hagiogr. lat. B. N. Parisiensis, 11, 1890, p. 464.

2. C'est donc encore nn livre qui doit être ajouté au groupe wisigothique détaillé par L. Delis le, Méianges de Paléographie et de Bibliographie, 1880, p. 53. J'en connais il est vrai, plusieurs autres.

3. J'ai lu seulement ceci : hic est liber dotisule (?). On aperçoit en outre une note wisigothique qui doit être contemporaine de la rédaction principale, bien que d'une autre main ; malheureusement, la lectnre est très incertaine: mater............... ancilla $x p i \mid x x$ annos auitat in anc celam. Le livre aurait donc appartenu d'abord à une moniale, abbesse plutôt que recluse.

4. Exempla si'ripturae visigoticae, 1883; à rapprocher surtout le $\mathrm{n}^{\circ} \mathrm{xIx}$ (Tolet. 10,25 de Madrid) de l'an 902.

5. Il y a en outre. deux lacunes dans le livre XI. entre les feuillets 86 et 87 , et les feuillets 88 et 89 . Tonte cette partie (cahiers XII, XIII et XIV) a été bouleversée ; il faut lire à la suite : $83-86,77-81,87-91,82,92-99$.

6. Cf. L. Delisle, Intentaire des manuscrits de la Bibliothèque Nationale: fonds de Cluni, 1884, p. 204. 
et ses compléments: les Dialogues de saint Grégoire, l'Histoire Lausiaque fondue avec l'Historia Monachorum, et les Vies des plus illustres moines, saint Antoine, saint Pachôme etc. I $\mathrm{Ce}$ " corpus , en effet, doit être démembré pour être intelligible. Les $A$ dhortationes, qui en forment le centre (p. 390-636), donnent juste le moyen de contrôler la collection wisigothique. Le manuscrit suivant rend le même service, conjointement.

(3.) Paris B. N. 5564, fol. 7-I 28 : manuscrit de Saint-Martial de Limoges, composé au XIIe siècle. L'accord de ce témoin avec les deux précédents est parfait, détails à part.

Nous tenons donc la longue série des vingt-trois livres, bien assurée par le concours des manuscrits de la Bibliothèque Nationale. La publication de Rosweyde garde sa valeur pratique, puisque nous retrouvons aussi, sans changement notable, la traduction latine faite à Rome, vers le milieu du VIe siècle, par le diacre Pélage et le sous-diacre Jean, - les mêmes personnages, en effet, qui devinrent papes l'un après l'autre et sont connus dans l'histoire comme Pélage II (555-560) et Jean III (560-563). Mais le travail de ceux-ci apparaît désormais dans sa continuité et sans cassure (livres I-XXI); et il se présente achevé, avec deux suppléments (livres XXII et XXIII), qui sont, en toute vraisemblance, l'œuvre d'un rédacteur contemporain, soucieux de communiquer entièrement au monde latin la tradition grecque qu'on connaissait à Rome. Si ces suppléments ont été omis le plus souvent au cours du moyen âge, on devine que c'est surtout la faute de l'explicit qui marquait la part des deux principaux traducteurs.

Ce simple raisonnement est soutenu, premièrement, par la présence des Sentences (livre XXIII) au terme des extraits qui composent la collection de Bobbio. On atteint de la sorte le commen. cement du VIII ${ }^{e}$ siècle, et la marge n'importe plus qui séparait la collection wisigothique de la version romaine. Parallèlement, la collection de Bobbio, en omettant les Correctiones (livre XXII), manifeste son caractère de recension abrégée; cette omission demeure sans conséquence, auprès de l'assurance obtenue que les Sentences font partie de l'ancien recueil.

En second lieu, le témoignage des manuscrits grecs constitue un argument invincible. Il est vrai, ceux que j'ai pu interroger (une douzaine $)^{2}$ sont très divers et, pour la plupart, plus complexes

1. Voir le détail $i b .$, p. 200 ss. (n० 114).

2. Bibliothèque Nationale $G r .914$, XIre siècle; $916, \mathrm{XIe}^{\mathrm{e}}$ s. ; $917, \mathrm{XII}{ }^{\circ}$ s.; 1596. XIe s. ; 1598, de l'année $993 ; 1600$, XIe s.; - Coisl. $126, \mathrm{X}^{\mathrm{e}} \mathrm{X} \mathrm{X}^{\mathrm{c}}$ s. ; $127, \mathrm{XI}^{\mathrm{e}}$ s.; 232 $\mathrm{XI}$ s.; $282, \mathrm{XI}^{\mathrm{e}} \mathrm{s}$; $283, \mathrm{XI} \mathrm{I}^{\mathrm{s}}$. Il serait trop long d'en faire ici l'analyse. 
que le texte latin ${ }^{x}$. Mais, les indications de Photius aidant ${ }^{2}$, on parvient à les départager et ramener à l'unité. Le recueil grec commun qui se cache derrière les manuscrits du moyen âge byzantin pourrait être ainsi décrit :

$I^{\circ}$ vingt groupes d'apophtegmes sur les différents aspects de l'ascèse monastique (livres I-XX du recueil latin);

$2^{\circ}$ un appendice du vingtième groupe mettant en scène douze anachorètes et rapportant leurs observances spirituelles (livre XXII du recueil latin);

$3^{\circ}$ les brefs apophtegmes anonymes des ascètes émérites (livre XXIII du recueil latin);

$4^{\circ}$ un questionnaire sans équivalent en latin, intitulé : $\Delta: \alpha \lambda \varepsilon \xi_{\xi \varepsilon, 5}$

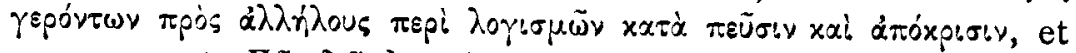

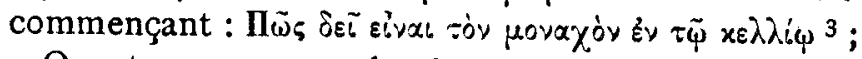

Quant aux « sept chapitres » de l'abbé Moïse (livre XXI du recueil latin), ils appartiennent sans aucun doute à la tradition grecque, mais en qualité d'élément secondaire et surajouté. Photius ne les mentionne pas; et de fait, trois manuscrits seulement de la Bibliothèque Nationale leur accordent une place, variable, parmi les autres apophtegmes ${ }^{4}$. De plus, ces manuscrits ne connaissent justement, sous le titre donné, que les $\S \mathrm{I}-7$, toute la suite fixe du latin ( $\$ 8-37)$ étant réellement un appendice composé de morceaux divers qui débordent l'annonce du titre 5 .

1. Par exemple, le lirre XX, correspondant à Rosweyde VT-3 (P. L, LXXIII, 1004-1014), offre cette suite dans les manuscrits 914 et $1600: \$ 1$ du latin; $\$ 3 ; \S 2$

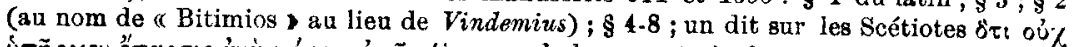

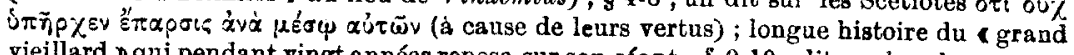
vieillard qui pendant vingt années reposa sur son séant; \$ 9-10; dit sur les observances diurnes ot nocturnes d'un habitant des $x^{\prime} \lambda_{\lambda} \lambda_{\iota \alpha} ; \$ 11-12$; longue bistoire sur l'ordination d'un évâque par l'archerêque ; sur quoi, l'histoire des douze anachorètes (livre XXII du recueil latin).

2. Noter bien, comme il a été indiqué précédemment, que Photius a aauté le troisième titre, ou ne l'a pas trouvé dans son volume.

3. Rosweyde s'est mépris sur la nature de ce chapitre, qu'il crut devoir identifier a vec celuí des douze anachorètes (cf. P. L., LXXIII, 853, note $c$ ). On trouvera le vrai texte dans $G r .914$, fol. 147-150 ; 917, fol. 91-94;1598, fol. 188v $.190 ; 1600$, fol. 16-19v ; Coisl. 28\%, fol. $98^{\mathrm{v}}-100$, etc. Il subsiste en effet dans toutes les collections grecques et l'on ne aurait s'y tromper; Photius a rapporté le titre précis.

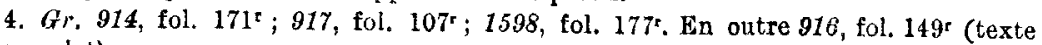
incomplet).

5. Les manuserits 914 et 917 insèrent, aussitôt après, un petit groupe analogue, sous

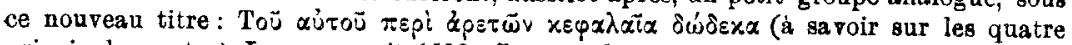
principales vertus). Le manuscrit 1598 offre une disposition plus intéressante. Il donne sans distinction, à la suite des sept chapitres une série particuliòre d'anecdotes qui permettent de comprendre l'arrangement du manuscrit grec dont dependait la recension latine. Les $\$ 8.37$ du livre XXI (Rosweyde VI-4) sont, Évidemment, un reliquat d'apophtegmes et d'historiettes erratiques qui n'araient pu être classes dans les vingt 
En résumé, le recueil latin en vingt-trois livres nous arrive complet grâce, à des manuscrits assez tardifs dont le plus ancien est une collection wisigothique qui pourrait remonter au dernier quart du IX' siècle. Cependant, quelques manuscrits fragmentaires ou abrégés font connaitre plus tôt des portions de ce même recueil, et finalement l'on est autorisé à croire qu'il fut formé, tel qu'on le possède encore, par des membres du clergé romain vers le milieu du VI ${ }^{e}$ siècle. Ces traducteurs ont disposé d'une excellente documentation, et leur œuvre differe peu, somme toute et quant à l'aspect général, de l'ensemble des manuscrits du moyen âge byzantin. De part et d'autre, on a vingt livres, un peu plus chargés de matière - c'est-à-dire interpolés - dans la tradition grecque directe. Ensuite, on se trouve en face de deux suppléments parallèles qui ne coincident pas absolument ; mais c'est surtout une question d'arrangement des parties. Un tableau fera mieux ressortir les ressemblances et les dissemblances que décèle l'analyse.

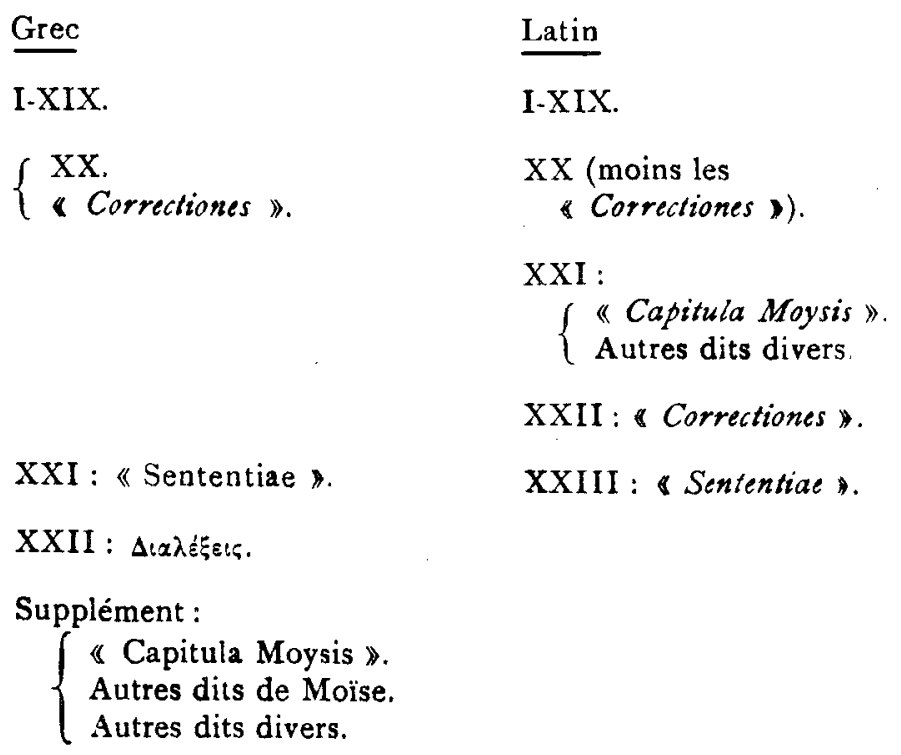

On voit clairement que les deux colonnes contiennent à peu près la même matière, différemment distribuée. Le traducteur latin qui a repris la plume après le sous-diacre Jean, pour parfaire le travail de celui-ci, se sera contenté de recueillir quelques éléments tradi-

livres réguliers. Car-tous ces articles proviennent du fonds grec, et on les retroure çà et là dans les représentants de cette tradition; je me propose précisément de traiter ailleurs du $\$ 8$ (sur les sept ordres du Christ), qui a joui d'une longue fortune en Occident. 
tionnels laissés de côté, intentionnellement ou non, par son prédécesseur. Ce faisant et sans modifier le plan déjà réalisé, il ne s'est guère éloigné des modèles grecs, à tel point que le grand recueil latin des apophtegmes est maintenant, en raison de sa date, un témoin de premier ordre pour qui veut apprécier cette curieuse littérature $\mathbf{x}$

Il reste à lire la rédaction latine qui a été le point de départ de cette enquête.Les quatre manuscrits qui servent à en établir la lettre ont été présentés ci-dessus :

$$
\begin{aligned}
& B: \text { collection de Bobbio, fol. } 149^{\nabla}-151^{\gamma^{2}} \text {; } \\
& C: \text { collection de Cluny, p. } 634-636 ; \\
& M: \text { collection de Saint-Martial, fol. } 128 ; \\
& W: \text { collection wisigothique, fol. } 189 .
\end{aligned}
$$

A l'occasion, je devrai rappeler la leçon du grec: $G$. Il faut bien avouer que cette version n'est pas excellente, si on la met en regard de l'original : ni très élégante ni très précise ni très fidèle au sens, tout en affectant, parfois, un extrême littéralisme. Quant au texte lui-même, la comparaison des quatre témoins montre qu'il a un long passé. $C$ et $M$ ont un ascendant commun, mais qui doit être beaucoup plus ancien qu'eux. Il doit y avoir aussi quelque lien entre $M$ et $W . B$ est souvent fautif, plus que ses partenaires; en revanche, on lui doit de connaitre en latin les deux dernières sentences. Tout ceci nous ramène très haut, non loin de l'original. D'ailleurs, dans presque tous les cas, le contrôle du grec garantit la teneur du texte.

\section{INCIPIUNT SENTENTIAE PATRUM.}

1 Inquisitus senex cuius modi deberet esse monachus respondit: $\mathrm{Si}$ quantum in me est, solus ad solum.

2 Item inquisitus senex a quodam cur in heremo ambulans formidaret respondit : Quia adhuc uiuis.

3 Inquisitus senex quid ad saluandum fieri oporteret - erat autem senex plectens palmas nec eleuans oculos ab opere -- ait: Quod uides.

1. Les petits recueils qui portent les noms de Paschase et de Martin demandent une étude spéciale à partir des manuscrits; je me suis abstenu d'en parler pour ne pas embroujller la question.

2. Les particularités orthographiques de ce manuscrit seront négligées habituellement dans l'apparat ; $B$ epelle quur $c i$ pour $t i$, $\&$ pour $i$, u pour o etc. - Je dois la collation à la grande obligeance de Mgr Luigi Grammatica, préfet de l'A mbrosiana. 
Inquisitus senex quid causa esset quod numquam pusillanimis inueniretur respondit : Mortem in dies singulos operior.

Inquisitus a quodam senex cur frequenter pusillanimis esset respondit : Nondum terminum uidisti.

Inquisitus senex quod esset opus monachi respondit : Discretio.

Inquisitus senex : Vnde mihi fornicatione temptari ? - ait : Multo cibo et somno.

Inquisitus senex quid monachus facere deberet respondit : Omnium bonorum operationem et malorum abstinentiam.

Dicebant senes : Speculum monachi esse orationem.

Idem ipsi aiebant : Nihil esse deterius ab iudicando.

Dicebant idem : Pignus cogitationibus numquam esse dandum.

Addebant etiam : Coronam esse monachi humilitatem.

Referebant rursum: Omni superuenienti tibi cogitationi dic : « Noster es an aduersariorum? - et omnimodis confitetur.

Aiebant senes : Mens fons est ; si fodias, mundatur; si stercores, sordescit.

Quidam senex ait : Ego credo quia non est iniustus deus ut de custo dia tollat et in custodiam mittat.

Ait senex : In omnibus sibi uiolentum esse; hoc est iter dei.

Ait senex : Nihil ante fieri oportere quam inquiras conscientiam si propter deum fit quod facturus es.

Ait senex : Si monachus tunc tantum cum ad crandum stat orat, hic numquam orat.

Ait senex : Viginti anuis permansi unara obpugnans cogitationem, ut omnes homines sicut unum uideam.

Ait senex: Omnium virtutum discretionem esse permaximam.

Inquisitus senex unde anima possessionem humilitatis adquireret respondit: Sua tantum sollicite cogitando mala.

Ait senex: Sicut terra numquam deorsum cadit, ita nec humilitas decidit.

Ait senex : In his quae adprehendere potui numquam secundus fui.

Ait senex: Pudor est monacho omnia quae sua sunt relinquere, peregrinari pro domino, et post haec ad poenam ire.

Ait senex : Generatio ista non quaerit hodie sed crastinum.

Dicebant senes; Opus nostrum concremare est ligna.

Ait senex : Noli esse cogitans.

Ait senex : Non irascitur humilitas nec inritat aliquem.

Ait iterum senex : Sedere bene in cellula cumulat bonis.

Ait senex : Vae homini quando nomen ipsius maius est quam operationes eius.

Ait senex: Fiducia et risus cannetum consumenti igni sunt similes.

Ait senex : Qui sibi propter deum riolentus est homini exaequabitur confessori.

Idem ait: Frouk quis stultus pro domino factus fuerit sapiens efficietur a domino. 

inspectio.

Ait senex : Haec dominus requirit ab homine : mentem, uerbum et operationem.

Idem ait: Haec sunt quibus homo indiget: timere iudicium dei, odio habere peccata, diligere uirtutem et deprecari dominum semper.

Cui est honor, gloria et imperium in saecula saeculorum amen.

Titre : sent. sanctorum p. C.

I. senes $\mathrm{B}$, et encore plus loin quelquefois. monachum $\mathrm{C}$ guanto $\mathrm{C}^{\mathbf{1}}$. in : ad $\mathrm{B}$ omis par $\mathbf{M}$.

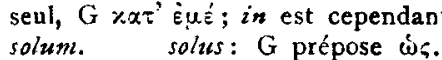
toujours le tour personnel: si: sicut $\mathrm{C}^{2}$

probable, à cause du voisinage de ad ( $\pi$ pó) suos. opere: $\mathrm{M}$ ajoute et. quo $\mathrm{B}$, boj $\dot{\omega} \mathrm{G}$ G. 4. causae $\mathrm{C}$. pusillanimis

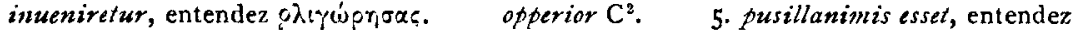

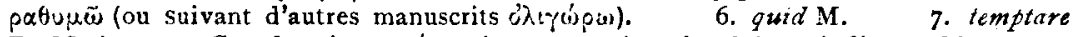
$B$; $M$ ajoute $e t, G$ a de même $x \alpha i$, mais cette conjonction lui est indispensable et tous

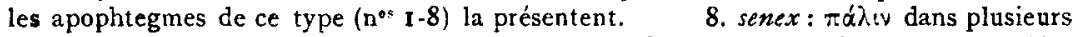
manuscrits. respondit omis par B. 10. agebant $\mathrm{B}$, et de même $\mathrm{I} 4$. abiudicando $\mathrm{C}$ adiudicando $\mathrm{M}$ adiudicandum $\mathrm{B} . \quad 13$. superutniente $\mathrm{B}$. noster (í,uetépos,

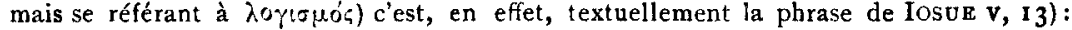
nostra C. omnimodis $(\pi \dot{x}, \tau \omega s)$. confitetur: óuojorriose. I4. est: et $B$ stercores : $\pi 00 \sigma$ (wors (il faut entendre : si tu apportes des terres). sor-

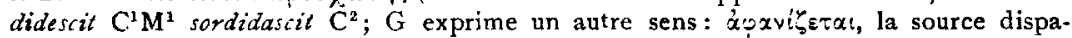
rait. 15. custodiamittat B. 16. omnis B. violentiam $\mathrm{M}$. dei: le recueil de Martin, qui offre une autre traduction de la même sentence, ajoute : et opus monachi (P. L., LXXIV, 392, $\mathrm{n}^{\circ}$ 92). I7. conscientiam (xapoi $(\alpha \mathrm{y})$ : consequenciam B. 18. senex: $\pi \dot{\alpha} \lambda \downarrow v$ dans plusieurs manuscrits. adorandum $\mathrm{BCM}$. I9. $X X$ B. unam omis par CM. oppugnans $\mathrm{C}$. 2o. fer maximam $\mathrm{C}$ uel maxi. mam B. 21, animam $\mathrm{B}$. suam B. 22, humilitatem $\mathrm{B} \delta \tau \times \pi \varepsilon$ !v $\bar{\omega} \vee \mathrm{G}$. deci. det $\mathrm{B}$. 23. in id quod $\mathrm{B}$, mais öax $\mathrm{G}$. appreh. CM. 24. monachi B. quae sua, avec ces mots commence le témoignage de $\mathrm{W}$ (le copiste a écrit exactement ques uas unt). peregrinare $B$. pro omis par $M$. Après cette sentence, les manuscrits grecs d'accord en inscrivent une autre que le rédacteur latin a oubliée ou n'a

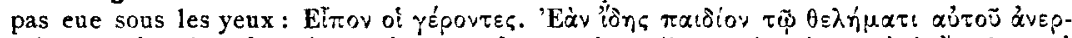

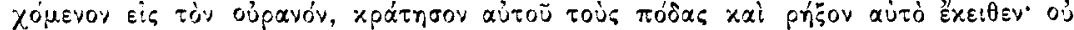

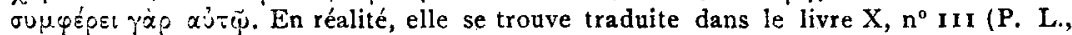
LXXI11, 932). 26. lignam B. 27. Cette sentence est omise par CM. cogi-

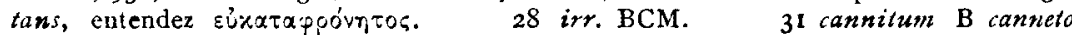
MW. consumens B. 32. homini omis par M, G fait suivre ce mot d'une ponc-

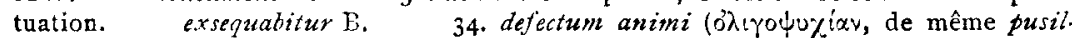
lanimitatem P. L., LXXIII, 1019 ; mais plusieurs manuscrits grees ont òiyopiay): de. fecto B. $\quad \mathrm{CMW}$ prennent fin avec cette sentence; $\mathrm{CM}$ ajoutent Expliciunt sententiae de diuersis patribus collectue, W simplement Explicit feliciter deo gratias. $\quad 35$. domi. nus: $\delta$ Ucos. hominem $\mathrm{B}$. 36. G lie chaque membre par $\%$ al. peccata.

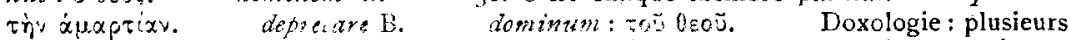

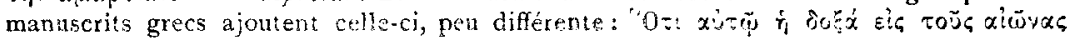
àuriv. 